\title{
Multimorbidity Analysis of 13 Systemic Diseases in Northeast China
}

\author{
Jianxing Yu ${ }^{1}$, Fangying Song ${ }^{1}$, Yingying Li ${ }^{1}$, Zhou Zheng ${ }^{1}$, Huanhuan Jia ${ }^{1}$, Yuzhe Sun ${ }^{1}$, \\ Lina Jin $^{2}$ and Xihe $Y u^{1, *}$ \\ 1 Social Medicine and Health Service Management, School of Public Health, No. 1163 Xinmin Street, \\ Jilin University, Changchun 130021, Jilin, China; yjxjlu@163.com (J.Y.); a15703410189@126.com (F.S.); \\ jdgwlyy@163.com (Y.L.); zhengzhou19@mails.jlu.edu.cn (Z.Z.); jhh_1994@163.com (H.J.); \\ yuzhe35789@163.com (Y.S.) \\ 2 Epidemiology and Biostatistics, School of Public Health, No. 1163 Xinmin Street, Jilin University, \\ Changchun 130021, Jilin, China; jinln@jlu.edu.cn \\ * Correspondence: xhyu@jlu.edu.cn
}

Received: 24 January 2020; Accepted: 8 March 2020; Published: 11 March 2020

Abstract: Background: Multimorbidity not only affects the quality of patients' lives, but can also bring a heavy economic burden to individuals, families and society. The purpose of this study was to reveal the connections between diseases, especially the important role each disease played in the entire multimorbidity network. Methods: A total of 1,155,734 inpatients were enrolled through multistage stratified random sampling in Jilin Province in 2017. Categorical variables were compared using the Rao-Scott- $\chi 2$ test. Weighted networks were adopted to present the complex relationships of multimorbidity. Results: The distributions of the number of diseases differed significantly by gender, age and health insurance scheme $(P<0.001)$. Diseases of the respiratory system had the highest weight in multimorbidity in young people. Endocrine, nutritional and metabolic diseases and circulatory system diseases were often associated with other systemic diseases in middle aged and old people. Conclusions: Multimorbidity with respiratory system diseases in young people should not be overlooked. Additionally, effective prevention efforts that target endocrine, nutritional and metabolic diseases and circulatory system diseases are needed in middle aged and old people.

Keywords: multimorbidity; systemic diseases; weighted networks

\section{Introduction}

Multimorbidity is defined as the presence of more than one health condition in an individual [1-4]. A person with numerous long-term health conditions has an increased risk of depression, hospitalizations, polypharmacy, loss of physical functioning, and even premature death $[5,6]$. In addition, multimorbidity places a substantial economic burden on the healthcare system [7-10], especially in the world's most populous country, China [11,12], because patients with multimorbidity often require additional intensive treatment and monitoring by nurses, physicians and other healthcare staff $[13,14]$. Therefore, the identification of the key determinants of multimorbidity is an important public health aim that should be addressed urgently.

To date, most studies have focused on single diseases while ignoring multimorbidity [15-17]. Some studies on the prevalence of multimorbidity did not consider the significance of the connection between diseases $[18,19]$. According to a literature review, most of the recent studies were based on self-reporting of prevalence by specific populations in specific environments, and the sample size was not large enough. For example, Alessandra's study [2] involved only 1099 elderly participants in the Kungsholmen Project, and they explored the role of age, gender, and socioeconomic status in the occurrence of chronic diseases and multimorbidity. 
To the best of our knowledge, few studies have defined diseases based on the international standard (e.g., the International Statistical Classification of Diseases and Related Health Problems 10th Revision (ICD-10)). The ICD can be used not only to increase the accuracy of disease differentiation but also to monitor the incidence and prevalence of diseases and other health problems, providing a picture of the general health situation of populations. To facilitate a visual understanding of 13 systemic disease combinations, this study created a multimorbidity network graph.

\section{Methods}

\subsection{Study Population}

A large-scale cross-sectional survey was implemented among people who were hospitalized in 20 general hospitals in Jilin Province in 2017. A total sample size of 1,300,683 inpatients was obtained through multistage stratified random sampling. The measured variables were socio-demographics (such as age, gender and health insurance scheme), disease names and ICD-10 classifications. In this study, only 13 systemic diseases were included, and the inpatients with some diseases and conditions (such as certain infectious or parasitic diseases, pregnancy, childbirth and puerperium) were excluded because these diseases were infectious diseases, congenital diseases or other conditions (see the details in Part 1 of the online Supplementary Materials). In the end, this study explored multimorbidity in $1,155,734$ inpatients.

\subsection{Ethics Statement}

The hospitals in the study informed the participants in advance that their disease information would be used for research, but participant names and any other personalized information were not collected. All research methods followed the guidelines of investigation, and written informed consent was obtained from all of the participants before data collection. All participants consented to this study, including the parents/legal guardians of those under the age of 18 . This study was approved by the ethics committee of the School of Public Health, Jilin University. All procedures involving human participants complied with the ethical standards of the institutional and national research committees.

\subsection{Data Collection}

The target sample was obtained through multistage stratified random sampling. First, considering comprehensive analyses of geographical locations, economic development levels and the health service statuses of each region, Changchun, Jilin City, Tonghua, Baicheng and Yanbian were selected for inclusion. Then, one district and two counties in each administrative region were selected. Finally, on the basis of the administrative region, a total of 20 general hospitals were selected as the monitoring institutions in this study.

We included all consecutive inpatients, and the dataset consisted of data regarding age, gender, disease name, ICD-10 classification, and health insurance scheme from medical records. To improve the accuracy of the data, we recruited a professional team to organize the data. A total of 20 interviewers with medical backgrounds and proficiency in EXCEL were recruited and uniformly trained to check basic information (such as age, gender, and health insurance). In addition, a panel of 30 general practitioners with more than 3 years of work experience verified that the disease names matched the ICD-10 classifications.

The survey was implemented by the School of Public Health, Jilin University in Jilin Province in 2017. According to relevant regulations, we are sorry that the data cannot be shared.

\subsection{Statistical Analyses}

Categorical variables were expressed as counts or percentages and compared using the Rao-Scott- $\chi^{2}$ test. Weighted networks were adopted to present the complex relationships of multimorbidity. The nodes of the network represented the systemic diseases, and the size of each 
systemic disease node was the weight as it occurred together with all other diseases. The thickness of each line connecting two systemic disease nodes was the degree of association between the two systemic diseases. Degree was the number of nodes that a focal node was connected to, which measured the involvement of the node in the network. Network density and average degree were used to evaluate the sparsity of a network. The network density of an undirected graph with $\mathrm{N}$ nodes and $\mathrm{M}$ edges was defined as $2 \mathrm{M} / \mathrm{N}(\mathrm{N}-1)$, which described the portion of the potential connections $(\mathrm{N}(\mathrm{N}-1) / 2)$ in a network that were actual connections $(\mathrm{M})$. The average degree was defined as the average of the degrees of all nodes. The larger the network density (or average degree), the denser the network [20,21]. All statistical analyses were performed with R version 3.6.1 (University of Auckland, Oakland, New Zealand). Statistical significance was set at a $P$ value $<0.05$.

\section{Results}

We analyzed data from 1,155,734 patients from 20 general hospitals in Jilin Province. The subjects were divided into three groups according to the number of diseases (see details in Table 1). The distribution of the number of diseases differed significantly by gender, age and health insurance scheme $(P<0.001)$. In addition, the number of male patients was higher than that of female patients, and the proportion of male patients with two or more diseases was higher than the proportion of female patients with two or more diseases. Overall, the proportion of patients suffering from one disease decreased with age, while the proportion of patients suffering from three or more diseases increased. In terms of health insurance schemes, the proportion of patients with one disease with New-Type Rural Cooperative Medical System (NRCMS) and Urban Social Pension Scheme (USPS) insurance was higher than that of patients with one disease with Urban Employment Basic Medical Insurance (UEBMI), Urban Resident Basic Medical Insurance (URBMI) and other insurance, but the trend in patients with two or more diseases was the opposite.

Table 1. Descriptive characteristics of the patients by the number of diseases.

\begin{tabular}{|c|c|c|c|c|c|c|}
\hline & n (\%) & 1 & 2 & $\geq 3$ & $x^{2}$ & $P$ \\
\hline $\begin{array}{c}\text { All } \\
\text { patients }\end{array}$ & $1,155,734(100 \%)$ & $598,120(51.75 \%)$ & $354,787(30.70 \%)$ & $202,827(17.55 \%)$ & & \\
\hline \multicolumn{7}{|l|}{ Gender } \\
\hline Male & $608,943(52.69 \%)$ & $297,967(48.93 \%)$ & $196,980(32.35 \%)$ & $113,996(18.72 \%)$ & \multirow{3}{*}{4125.02} & \multirow{3}{*}{$<0.001$} \\
\hline Female & $546,791(47.31 \%)$ & $300,153(54.89 \%)$ & $157,807(28.86 \%)$ & $88,831(16.25 \%)$ & & \\
\hline \multicolumn{5}{|l|}{$\begin{array}{c}\text { Age } \\
\text { (years) }\end{array}$} & & \\
\hline $0 \sim$ & $55,198(4.78 \%)$ & $37,385(67.73 \%)$ & $13,519(24.49 \%)$ & $4294(7.78 \%)$ & \multirow{5}{*}{$32,416.92$} & \multirow{5}{*}{$<0.001$} \\
\hline $18 \sim$ & $116,520(10.08 \%)$ & $80,048(68.70 \%)$ & $26,167(22.46 \%)$ & $10,305(8.84 \%)$ & & \\
\hline $45 \sim$ & $420,757(36.41 \%)$ & $218,663(51.97 \%)$ & $128,460(30.53 \%)$ & $73,634(17.50 \%)$ & & \\
\hline $65 \sim$ & $466,980(40.41 \%)$ & $226,908(48.59 \%)$ & $146,190(31.31 \%)$ & $93,882(20.10 \%)$ & & \\
\hline $85 \sim$ & $96,279(8.33 \%)$ & $35,116(36.47 \%)$ & $40,451(42.01 \%)$ & $20,712(21.51 \%)$ & & \\
\hline \multicolumn{7}{|c|}{ Health insurance schemes } \\
\hline UEBMI & $521,337(45.11 \%)$ & $259,243(49.73 \%)$ & $157,185(30.15 \%)$ & $104,909(20.12 \%)$ & \multirow{5}{*}{$24,816.04$} & \multirow{5}{*}{$<0.001$} \\
\hline URBMI & $248,988(21.54 \%)$ & $119,751(48.10 \%)$ & $82,401(33.09 \%)$ & $46,836(18.81 \%)$ & & \\
\hline NRCMS & $197,144(17.06 \%)$ & $116,834(59.26 \%)$ & $54,593(27.69 \%)$ & $25,717(13.04 \%)$ & & \\
\hline USP & $151,632(13.12 \%)$ & $92,223(60.82 \%)$ & $44,127(29.10 \%)$ & $15,282(10.08 \%)$ & & \\
\hline Others * & $36,633(3.17 \%)$ & $10,069(27.49 \%)$ & $16,481(44.99 \%)$ & $10,083(27.52 \%)$ & & \\
\hline
\end{tabular}

UEBMI: Urban Employee Basic Medical Insurance; URBMI: Urban Resident Basic Medical Insurance; NRCMS: New Rural Cooperative Medical Scheme; USP: uninsured patients; * "others" included commercial health insurance, work-related injury insurance, maternity and medical assistance.

Figures 1 and 2 show the proportions of the numbers of diseases by age for males and females. The proportion of the number of diseases decreased in those 0 to 19 years old, increased in those 20 to 64 years old, and then decreased slowly in those older than 65 years in both males and females. In addition, the proportion of three or more diseases was the lowest and the proportion of one disease was the highest in those less than 55 years old. However, the proportion of three or more diseases was the highest in those 55 to 84 years old. Figures 1 and 2 also show that the age group with the 
lowest proportion and the age group with the highest proportion were the 10 19 and 60 69 years age groups, respectively.

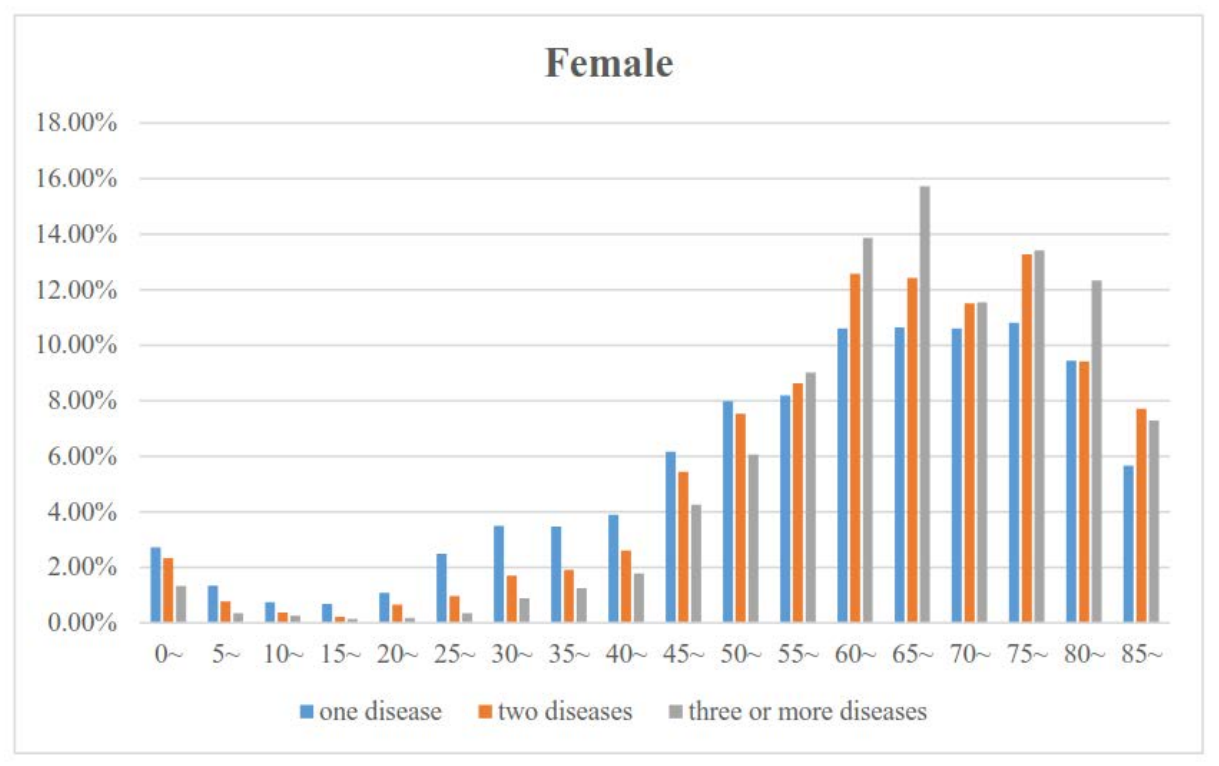

Figure 1. Association between age and the proportion of the number of diseases for females.

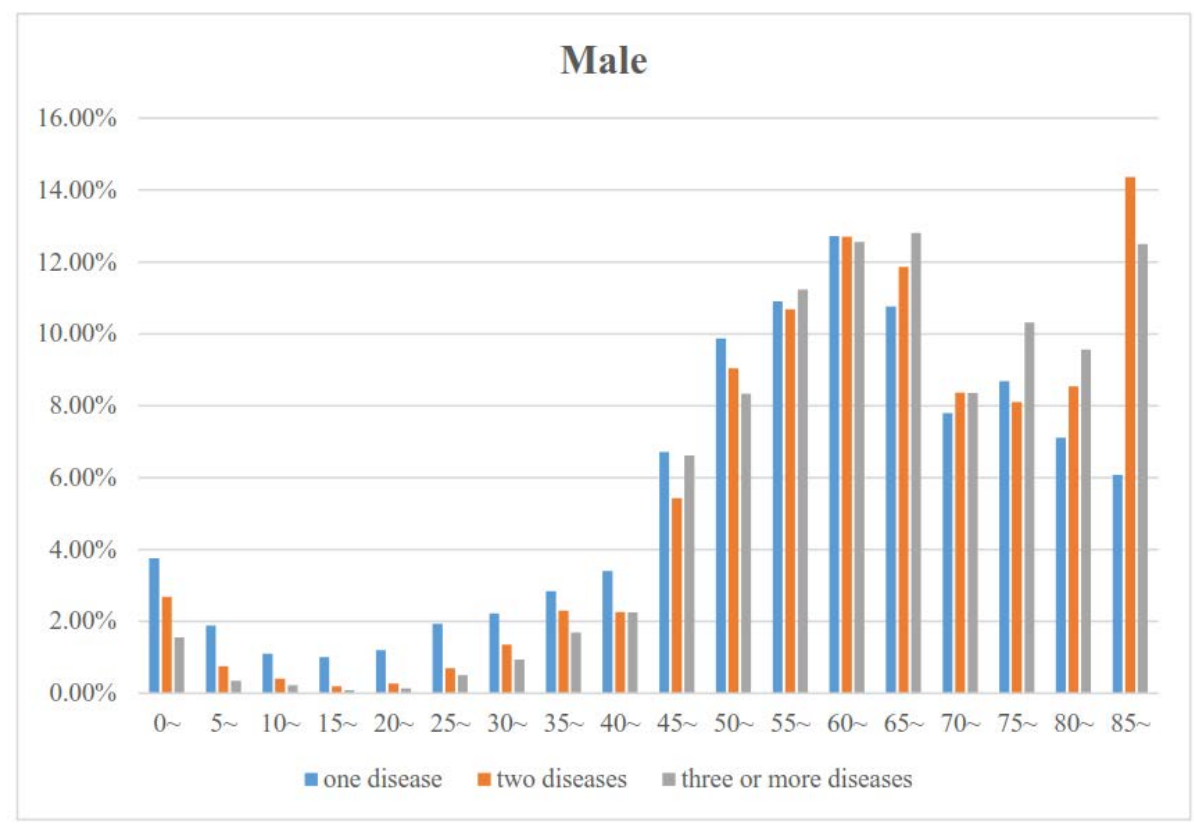

Figure 2. Association between age and the proportion of the number of diseases for male.

Table 2 shows the abbreviation of the 13 systemic diseases. The proportions of the ten most common two-disease combinations in the study population are presented in Table 3. In particular, endocrine, nutritional and metabolic diseases (ENM) and diseases of the circulatory system (CirS) had a relatively high proportion of combinations. Figure 3 shows a visual understanding of all the disease combinations. Endocrine, nutritional and metabolic diseases (ENM) and diseases of the circulatory system (CirS) had notably high weights in both males and females (illustrated by the sizes of the nodes) and frequently occurred together with other diseases (illustrated by the thickness of the lines connected to these nodes). 
Table 2. The Abbreviation of the 13 systemic diseases.

\begin{tabular}{cc}
\hline The Name of the Systemic Diseases & Abbreviation \\
\hline Neoplasms & Neo \\
Diseases of the blood and blood-forming organs and & BBI \\
certain disorders involving the immune mechanism & ENM \\
Endocrine, nutritional and metabolic diseases & MB \\
Mental and behavioral disorders & NerS \\
Diseases of the nervous system & EA \\
Diseases of the eye and adnexa & EM \\
Diseases of the ear and mastoid process & CirS \\
Diseases of the circulatory system & ResS \\
Diseases of the respiratory system & DigS \\
Diseases of the digestive system & SkSt \\
Diseases of the skin and subcutaneous tissue & MuSC \\
Diseases of the musculoskeletal system and & GenS \\
connective tissue & \\
Diseases of the genitourinary system &
\end{tabular}

Table 3. The proportions of the ten most common two-disease combinations by gender.

\begin{tabular}{ccc}
\hline & Men & Women \\
\hline 1 & ENM-CirS $(18.50 \%)$ & ENM-CirS $(16.73 \%)$ \\
2 & CirS-ResS $(13.37 \%)$ & CirS-ResS $(9.08 \%)$ \\
3 & ENM-ResS $(6.06 \%)$ & CirS-GenS $(5.87 \%)$ \\
4 & NerS-CirS $(5.22 \%)$ & CirS-DigS $(5.28 \%)$ \\
5 & CirS-DigS $(5.05 \%)$ & ENM-ResS $(5.07 \%)$ \\
6 & ENM-NerS $(4.77 \%)$ & NerS-CirS $(4.46 \%)$ \\
7 & ENM-DigS $(4.68 \%)$ & ENM-DigS $(4.41 \%)$ \\
8 & CirS-GenS $(4.29 \%)$ & ENM-GenS $(4.23 \%)$ \\
9 & ENM-GenS (3.87\%) & BBI-GenS $(4.22 \%)$ \\
10 & ResS-DigS (3.35\%) & ENM-NerS $(3.90 \%)$ \\
\hline
\end{tabular}
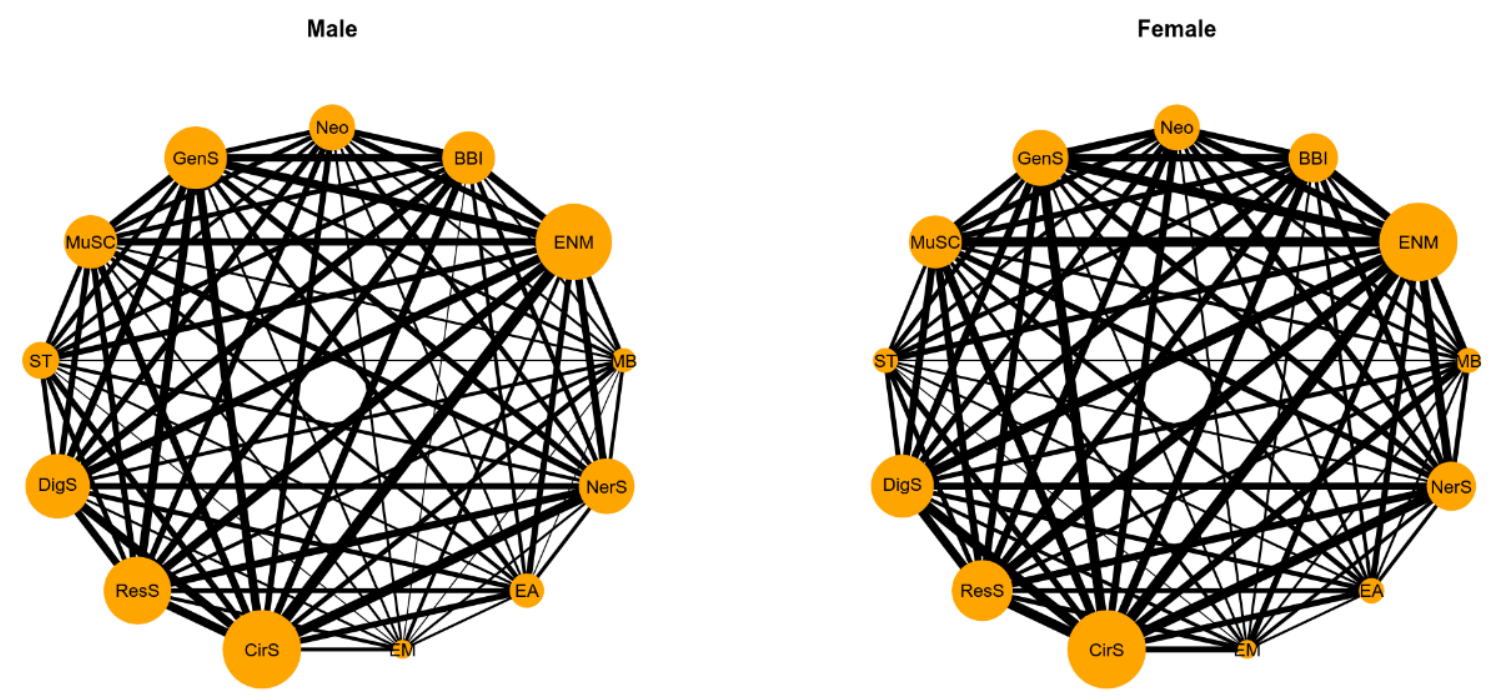

Figure 3. Multimorbidity networks by gender for 13 systemic diseases.

Figure 4 shows the multimorbidity networks by age for 13 systemic diseases. Generally, the multimorbidity networks were different for different age groups. In the age group 0 17 years, respiratory system diseases (ResS) had the highest weight. In addition, the "ResS-CirS", "ResS-DigS", and "ResS-BBI" had notably high connections in the multimorbidity networks, and the multimorbidity networks were similar among genders. However, endocrine, nutritional and metabolic diseases (ENM) 
had the highest weight, and the "ENM-CirS-ResS", "ENM-CirS-DigS", and "ENM-CirS-GenS" triangles had notably high connections in the multimorbidity networks in the age group $18 \sim 44$ years. In addition, the size of Neoplasms (Neo) and blood and blood-forming organs diseases and certain disorders involving the immune system (BBI) nodes in male patients was bigger than that in female patients. In the age groups 45 64 years and 65 84 years, endocrine, nutritional and metabolic diseases (ENM) and circulatory system diseases (CirS) had the highest weights. Similar to the age group 18 to 44 years, the "ENM-CirS-ResS", "ENM-CirS-DigS", and "ENM-CirS-GenS" triangles had notably high connections in the multimorbidity networks. Notably, the average degrees of the connections/triangles in the age groups 45 64 years and 65 84 years were much higher than those in the age groups 0 17 years and 18 44 years. Finally, circulatory system diseases (CirS) had the highest weight in the group aged 85 years old and above, but the "ENM-CirS-ResS", "ENM-CirS-DigS", and "ENM-CirS-GenS" triangles still had notably high connections in the multimorbidity networks (for the proportions of the ten most common two-disease combinations in different age groups, see online Supplementary Materials Tables S2 and S3).
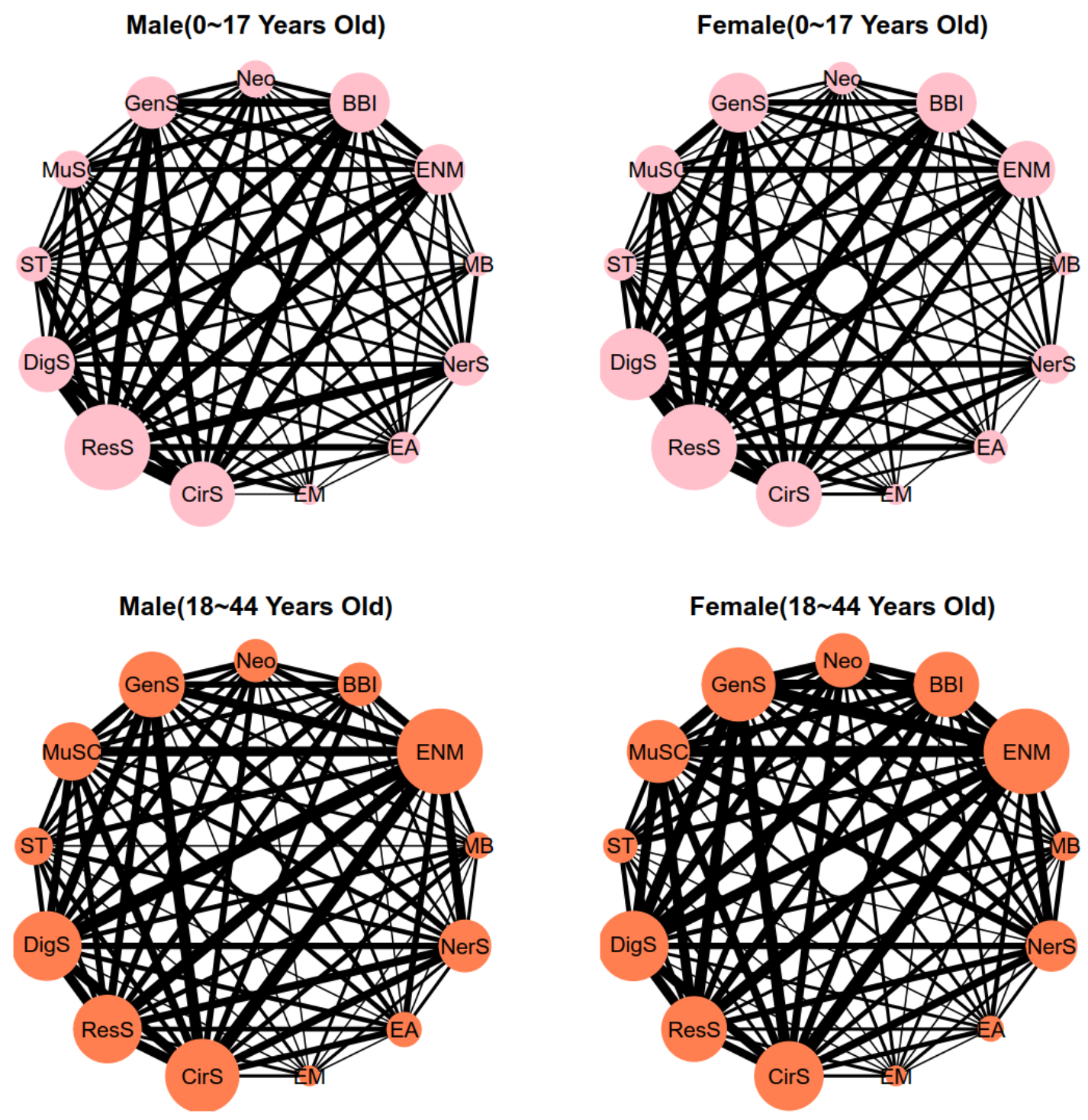

Figure 4. Cont. 

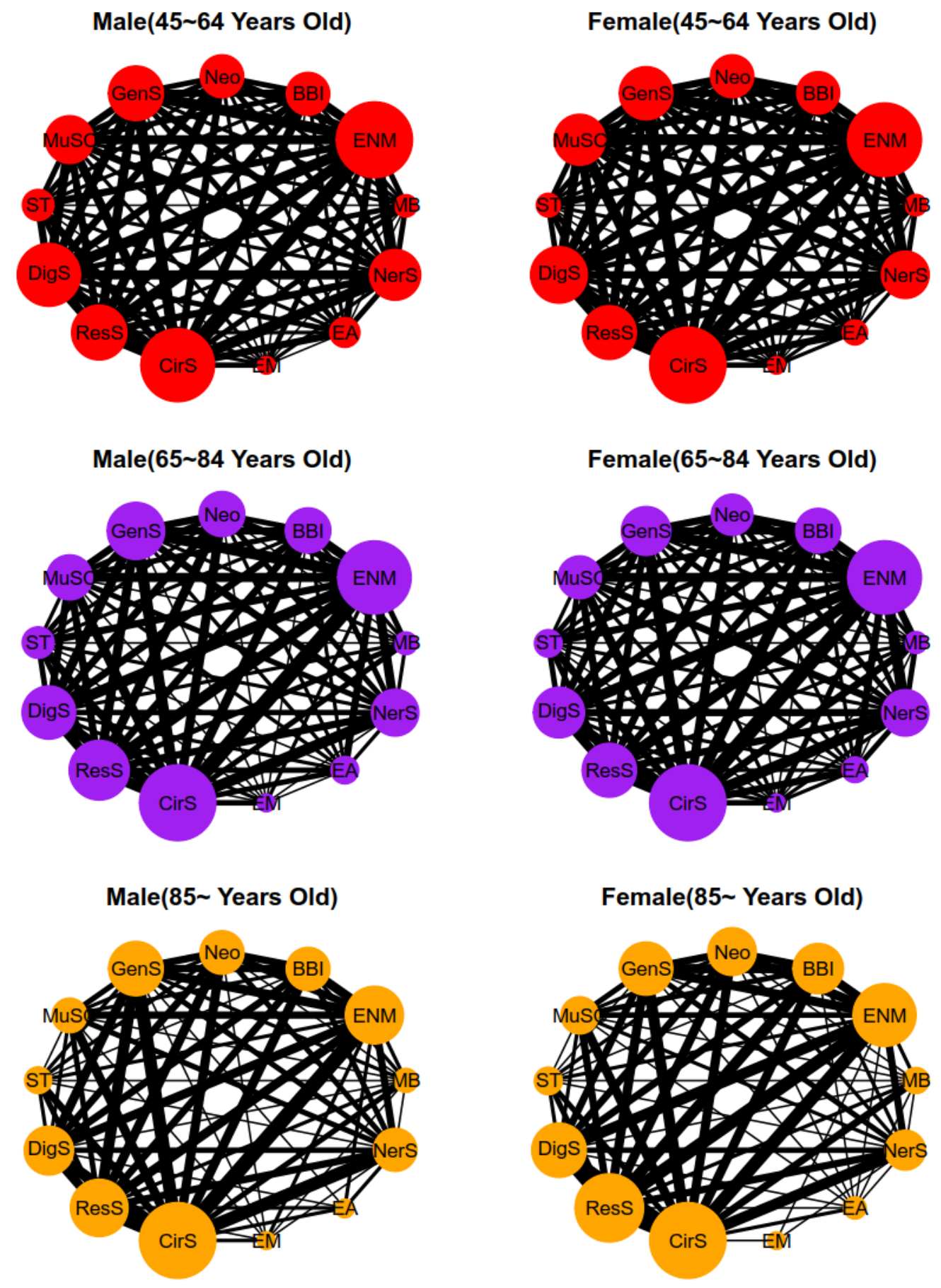

Figure 4. Multimorbidity networks by age group for 13 systemic diseases.

The multimorbidity networks were similar in the four kinds of health insurance schemes (except others), and ENM (endocrine, nutritional and metabolic diseases) and CirS (diseases of the circulatory system) had a relatively high proportion of combinations. Figure 5 shows a visual understanding of all the disease combinations. ENM (endocrine, nutritional and metabolic diseases) and CirS (diseases of the circulatory system) had notably high weights in UEBMI, URBMI and NRCMS and frequently occurred together with other diseases (for the proportions of the ten most common two-disease combinations in different age groups see online Supplementary Materials Table S4). 

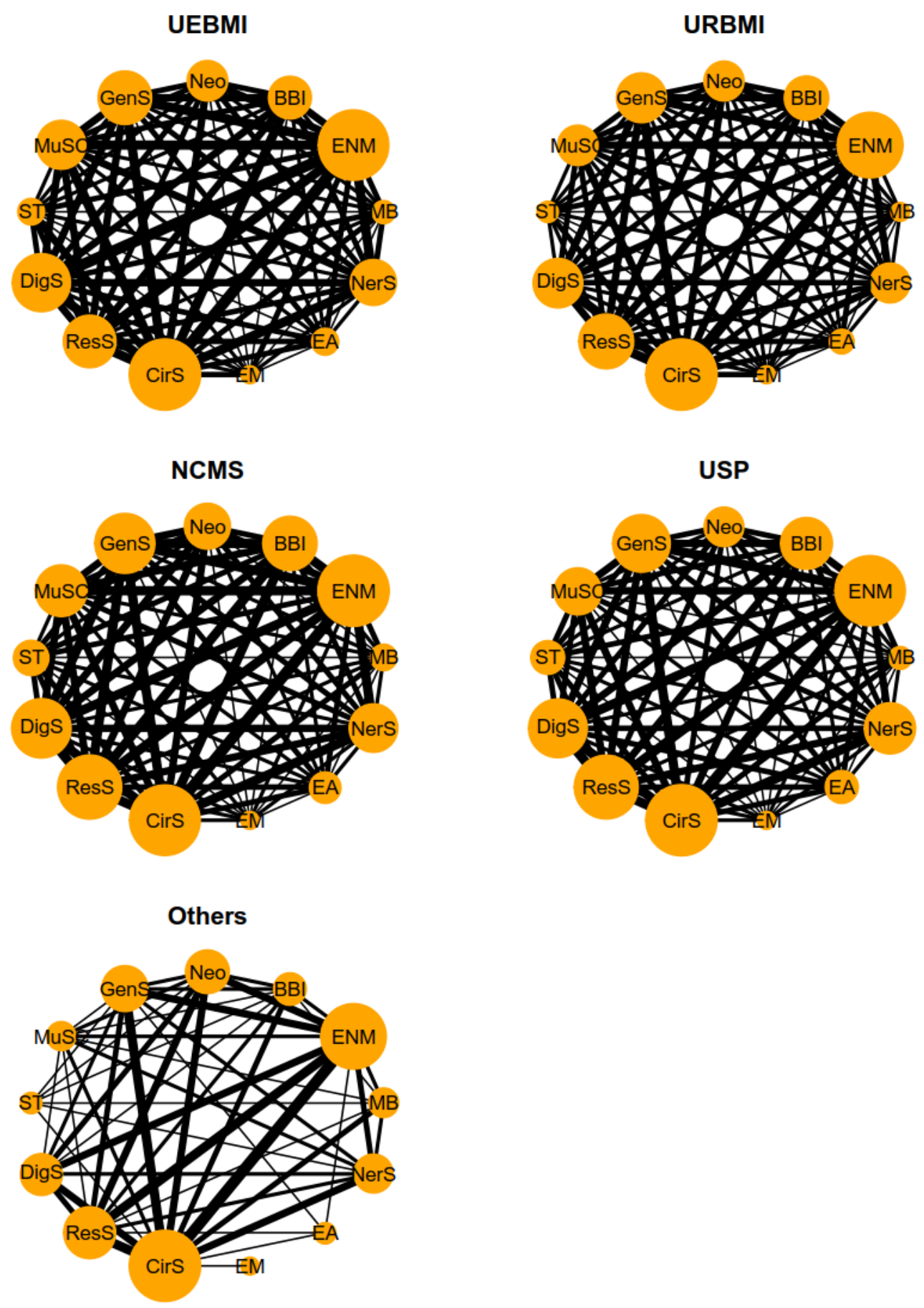

Figure 5. Multimorbidity networks by health insurance schemes for 13 systemic diseases.

\section{Discussion}

Multimorbidity is believed to bring great challenges to, and have important impacts on, current public health. Employing large cross-sectional data from a survey of Jilin Province, this study explored multimorbidity in hospitalized patients with regard to 13 systemic diseases, and examined the association of combinations comprising two diseases from these 13 systemic diseases. If we are to deal with an increasing prevalence of multimorbidity in an ageing population, we need to know about disease combinations so we can design best practice guidelines for clinicians [22]. In order to explore the combinations between systemic diseases, we created a network graph of the disease combinations, which facilitates a visual understanding of all combinations. 
Our analysis of a large representative dataset showed that multimorbidity was common. Out of all inpatients who had two or more diseases $48.25 \%$ were identified as having multimorbidity; this proportion was much higher than in other studies $[23,24]$. This result might be explained by the fact that our subjects were inpatients, as different study populations likely played a key role in the discrepancy $[25,26]$. However, this study focused on only inpatients in general hospitals. Inpatients with multiple disorders will have poor functional capacity, poor quality of life and extended hospital stays, and they need a general service. The need for several healthcare providers to treat multiple diseases across different medical specialties makes it difficult to provide a generalist service. A strong, generalist primary care team is the most obvious way to provide continuity, coordination, and, above all, individualized care for people with multimorbidity to minimize future disability and morbidity.

Furthermore, we found that the prevalence of multimorbidity was much higher in males than in females; this result might be ascribed to differences in body function, e.g., the life expectancy of females is 5 years longer than that of males [27]. Another possible explanation is the great differences in the habits and diets between genders; for example, males tend to consume alcohol, smoke and eat meat more often than females [28-30]. Further, endocrine, nutritional and metabolic diseases (ENM) and diseases of the circulatory system (CirS) had the highest weights in both males and females, but the "ENM-CirS" combination in males was relatively more prominent than that in females. It is suggested that the "ENM-CirS" combination be given more attention, especially in males.

The other notable finding was that the prevalence of multimorbidity was higher in old people $((146,190+93,882+40,451+20,712) / 557,614=54.02 \%)$ than in young or middle-aged people $(45.98 \%)$. This result agrees with results of previous work that showed that there was a substantial association between age and multimorbidity and that the prevalence of multimorbidity tends to be increased in older adults $[25,31,32]$. A possible reason for this might be that body immunity and function are greater in young people than in old people, so the proportion of the number of diseases was lowest in those 0 to 17 years old. In addition, respiratory system diseases (ResS) had the highest weight in multimorbidity in the age group $0 \sim 17$ years. This may be related to low respiratory immunity in children, large temperature differences in northeast China, and environmental pollution $[33,34]$. Thus, multimorbidity dominated by respiratory system diseases (ResS) should be the focus of attention given to young people.

The health challenges faced by adults are more complex than those faced by young people, as body immunity and function decline with age; therefore, research on adult multimorbidity cannot be overlooked. The proportion of the number of diseases increased in those 18 to 64 years old, and ENM (endocrine, nutritional and metabolic diseases) and CirS (circulatory system diseases) had higher weight than other systemic diseases in multimorbidity. Although those 18 to 64 years old had a denser multimorbidity network, the "ResS-CirS" combination in the age group 85 years or older was more marked than other combinations, where their CirS (circulatory system diseases) occupied a large percentage compared with that of those 18 to 64 years old. These findings suggest that different key prevention methods should be targeted towards different age groups.

Jilin Province has a temperate continental monsoon climate, and we speculate that inhabitants consume high amounts of animal fat and salt and few vegetables [35]. Additionally, the population at middle age is under substantial mental stress, which leads to people feeling run-down and ill. Notably, the proportion of three or more diseases was the highest in those aged 55 and older. Geriatricians play a significant role in the provision of care for the frailest elderly patients with multimorbidity, but in most countries, these patients are treated in primary care facilities regardless of the critical connections between different diseases.

Finally, health insurance schemes are a microcosm of different professions. However, we found that the multimorbidity networks of four kinds of health insurance schemes (excluding others) were similar, and the "CirS-ENM" combination was more marked than other combinations in the multimorbidity networks. This also indirectly explains the existence of certain internal multimorbidity correlations, which will not substantially change on the basis of gender, age and occupation. 
Some limitations should be noted here. On the one hand, only gender, age and health insurance scheme were investigated in the study, but other factors that might have effects on multimorbidity were worthy of further study. On the other hand, the participants in the study were inpatients, which might cause bias. Underlying these limitations is the lack of an internationally accepted standard for measuring multimorbidity. A clear conceptual framework must be developed that includes a consistent approach to the measurement of multimorbidity and age categorization in the study population to allow for comparison between studies and populations.

\section{Conclusions}

Different strategies should be separately developed to prevent multimorbidity in different age groups. Multimorbidity with respiratory system diseases in young people should not be overlooked. Additionally, effective prevention efforts that target endocrine, nutritional and metabolic diseases and circulatory system diseases are needed in middle aged and old people. Finally, knowledge of the common combinations of multimorbidity may help in planning the health services needed in the future, and the healthcare system requires further modifications to accurately identify and effectively manage patients with multimorbidity.

Supplementary Materials: The following are available online at http://www.mdpi.com/1660-4601/17/6/1817/s1. Table S1: International Statistical Classification of Diseases and Related Health Problems 10th Revision; Table S2: The proportions of ten most common two-disease combinations by age in males; Table S3: The proportions of ten most common two-disease combinations by age in females; Table S4: The proportions of ten most common two-disease combinations by health insurance scheme.

Author Contributions: J.Y. and X.Y. had the original idea for the study and carried out the design. X.Y. and F.S. provided valuable insight regarding the methodological approach and organization of the manuscript. J.Y., Y.L. and F.S. carried out the statistical analysis and reviewed the consistency of data included in the paper. J.Y., L.J. and F.S. drafted the manuscript. Z.Z., H.J., Y.S. and X.Y. revised the manuscript. All authors read and approved the final manuscript.

Acknowledgments: The authors gratefully acknowledge the financial support received from MOE (Ministry of Education in China) Project of Humanities and Social Sciences (Project No.18YJAZH118). We express our gratitude to the participants and colleagues who were involved in the study. The authors have no competing interests.

Conflicts of Interest: The authors declare no conflict of interest.

\section{References}

1. Barnett, K.; Mercer, S.W.; Norbury, M.; Watt, G.; Wyke, S.; Guthrie, B. Epidemiology of multimorbidity and implications for health care, research, and medical education: A cross-sectional study. Lancet 2012, 380, 37-43. [CrossRef]

2. Marengoni, A.; Winblad, B.; Karp, A.; Fratiglioni, L. Prevalence of chronic diseases and multimorbidity among the elderly population in Sweden. Am. J. Public Health 2008, 98, 1198-1200. [CrossRef] [PubMed]

3. Ng, S.K.; Tawiah, R.; Sawyer, M.; Scuffham, P. Patterns of multimorbid health conditions: A systematic review of analytical methods and comparison analysis. Int. J. Epidemiol. 2018, 47, 1687-1704. [CrossRef] [PubMed]

4. Prados-Torres, A.; Calderon-Larranaga, A.; Hancco-Saavedra, J.; Poblador-Plou, B.; van den Akker, M. Multimorbidity patterns: A systematic review. J. Clin. Epidemiol. 2014, 67, 254-266. [CrossRef] [PubMed]

5. Salisbury, C.; Man, M.S.; Bower, P.; Guthrie, B.; Chaplin, K.; Gaunt, D.M.; Brookes, S.; Fitzpatrick, B.; Gardner, C.; Hollinghurst, S.; et al. Management of multimorbidity using a patient-centred care model: A pragmatic cluster-randomised trial of the 3D approach. Lancet 2018, 392, 41-50. [CrossRef]

6. Kernick, D.; Chew-Graham, C.A.; O'Flynn, N. Clinical assessment and management of multimorbidity: NICE guideline. Br. J. Gen. Pract. 2017, 67, 235-236. [CrossRef]

7. Moffat, K.; Mercer, S.W. Challenges of managing people with multimorbidity in today's healthcare systems. BMC Fam. Pract. 2015, 16, 129. [CrossRef]

8. Hu, R.H.; Hsiao, F.Y.; Chen, L.J.; Huang, P.T.; Hsu, W.W. Increasing age- and gender-specific burden and complexity of multimorbidity in Taiwan, 2003-2013: A cross-sectional study based on nationwide claims data. BMJ Open 2019, 9, e28333. [CrossRef] 
9. Pefoyo, A.J.; Bronskill, S.E.; Gruneir, A.; Calzavara, A.; Thavorn, K.; Petrosyan, Y.; Maxwell, C.J.; Bai, Y.; Wodchis, W.P. The increasing burden and complexity of multimorbidity. BMC Public Health 2015, 15, 415. [CrossRef]

10. Laires, P.A.; Perelman, J. The current and projected burden of multimorbidity: A cross-sectional study in a Southern Europe population. Eur. J. Ageing 2019, 16, 181-192. [CrossRef]

11. Wang, S.B.; D'Arcy, C.; Yu, Y.Q.; Li, B.; Liu, Y.W.; Tao, Y.C.; Wu, Y.H.; Zhang, Q.Q.; Xu, Z.Q.; Fu, Y.L.; et al. Prevalence and patterns of multimorbidity in northeastern China: A cross-sectional study. Public Health 2015, 129, 1539-1546. [CrossRef] [PubMed]

12. Wang, H.H.; Wang, J.J.; Wong, S.Y.; Wong, M.C.; Li, F.J.; Wang, P.X.; Zhou, Z.H.; Zhu, C.Y.; Griffiths, S.M.; Mercer, S.W. Epidemiology of multimorbidity in China and implications for the healthcare system: Cross-sectional survey among 162,464 community household residents in southern China. BMC Med. 2014, 12, 188. [CrossRef] [PubMed]

13. Mori, T.; Hamada, S.; Yoshie, S.; Jeon, B.; Jin, X.; Takahashi, H.; Iijima, K.; Ishizaki, T.; Tamiya, N. The associations of multimorbidity with the sum of annual medical and long-term care expenditures in Japan. BMC Geriatr. 2019, 19, 69. [CrossRef] [PubMed]

14. Chen, W.; Safari, A.; FitzGerald, J.M.; Sin, D.D.; Tavakoli, H.; Sadatsafavi, M. Economic burden of multimorbidity in patients with severe asthma: A 20-year population-based study. Thorax 2019. [CrossRef] [PubMed]

15. Ye, J.; Li, Z.; Lv, Y.; An, L.; Yu, J.; Guo, X.; Yao, Y.; Yu, Y.; Jin, L. Associations of Blood Pressure with the Factors among Adults in Jilin Province: A Cross-Sectional Study Using Quantile Regression Analysis. Sci. Rep. 2017, 7. [CrossRef] [PubMed]

16. Liu, X.; Gu, W.; Li, Z.; Lei, H.; Li, G.; Huang, W. Hypertension prevalence, awareness, treatment, control, and associated factors in Southwest China: An update. J. Hypertens. 2017, 35, 637-644. [CrossRef] [PubMed]

17. Olack, B.; Wabwire-Mangen, F.; Smeeth, L.; Montgomery, J.M.; Kiwanuka, N.; Breiman, R.F. Risk factors of hypertension among adults aged 35-64 years living in an urban slum Nairobi, Kenya. BMC Public Health 2015, 15, 1251. [CrossRef]

18. Sakib, M.N.; Shooshtari, S.; St. John, P.; Menec, V. The prevalence of multimorbidity and associations with lifestyle factors among middle-aged Canadians: An analysis of Canadian Longitudinal Study on Aging data. BMC Public Health 2019, 19. [CrossRef]

19. Nguyen, H.; Manolova, G.; Daskalopoulou, C.; Vitoratou, S.; Prince, M.; Prina, A.M. Prevalence of multimorbidity in community settings: A systematic review and meta-analysis of observational studies. J. Comorb. 2019, 9, 2235042X-19870934X. [CrossRef]

20. Harding, A. Actor-Network-Theory and Micro-Learning Networks. Educ. Prim. Care 2017, 28, $295-296$. [CrossRef]

21. Jin, L.; Guo, X.; Dou, J.; Liu, B.; Wang, J.; Li, J.; Sun, M.; Sun, C.; Yu, Y.; Yao, Y. Multimorbidity Analysis According to Sex and Age towards Cardiovascular Diseases of Adults in Northeast China. Sci. Rep. 2018, 8, 8607. [CrossRef] [PubMed]

22. Starfield, B. Global health, equity, and primary care. J. Am. Board Fam. Med. 2007, 20, 511-513. [CrossRef] [PubMed]

23. Agborsangaya, C.B.; Ngwakongnwi, E.; Lahtinen, M.; Cooke, T.; Johnson, J.A. Multimorbidity prevalence in the general population: The role of obesity in chronic disease clustering. BMC Public Health 2013, 13, 1161. [CrossRef] [PubMed]

24. Quinaz, R.G.; Kislaya, I.; Salvador, M.R.; Cunha, G.S.; Nunes, B.; Dias, C. Multimorbidity in Portugal: Results from The First National Health Examination Survey. Acta Med. Port. 2019, 32, 30-37. [CrossRef]

25. Ishizaki, T.; Kobayashi, E.; Fukaya, T.; Takahashi, Y.; Shinkai, S.; Liang, J. Association of physical performance and self-rated health with multimorbidity among older adults: Results from a nationwide survey in Japan. Arch. Gerontol. Geriatr. 2019, 84, 103964. [CrossRef]

26. Dhalwani, N.N.; O’Donovan, G.; Zaccardi, F.; Hamer, M.; Yates, T.; Davies, M.; Khunti, K. Long terms trends of multimorbidity and association with physical activity in older English population. Int. J. Behav. Nutr. Phys. 2016, 13. [CrossRef]

27. World Health Organization. World Health Statistics 2015; WHO: Geneva, Switzerland, 2015.

28. Yu, J.; Tao, Y.; Dou, J.; Ye, J.; Yu, Y.; Jin, L. The dose-response analysis between BMI and common chronic diseases in northeast China. Sci. Rep. 2018, 8, 4228. [CrossRef] 
29. Muga, M.A.; Owili, P.O.; Hsu, C.Y.; Rau, H.H.; Chao, J.C. Association between Dietary Patterns and Cardiovascular Risk Factors among Middle-Aged and Elderly Adults in Taiwan: A Population-Based Study from 2003 to 2012. PLoS ONE 2016, 11, e157745. [CrossRef]

30. Park, K.; Son, J.; Jang, J.; Kang, R.; Chung, H.K.; Lee, K.W.; Lee, S.M.; Lim, H.; Shin, M.J. Unprocessed Meat Consumption and Incident Cardiovascular Diseases in Korean Adults: The Korean Genome and Epidemiology Study (KoGES). Nutrients 2017, 9, 498. [CrossRef]

31. Fried, T.R.; O'Leary, J.; Towle, V.; Goldstein, M.K.; Trentelange, M.; Martin, D.K. The effects of comorbidity on the benefits and harms of treatment for chronic disease: A systematic review. PLoS ONE 2014, 9, e112593. [CrossRef]

32. Lee, P.G.; Cigolle, C.; Blaum, C. The co-occurrence of chronic diseases and geriatric syndromes: The health and retirement study. J. Am. Geriatr. Soc. 2009, 57, 511-516. [CrossRef] [PubMed]

33. Yang, T.; Gbaguidi, A.; Yan, P.; Zhang, W.; Zhu, L.; Yao, X.; Wang, Z.; Chen, H. Model elucidating the sources and formation mechanisms of severe haze pollution over Northeast mega-city cluster in China. Environ. Pollut. 2017, 230, 692-700. [CrossRef] [PubMed]

34. Teng, B.; Zhang, X.; Yi, C.; Zhang, Y.; Ye, S.; Wang, Y.; Tong, D.; Lu, B. The Association between Ambient Air Pollution and Allergic Rhinitis: Further Epidemiological Evidence from Changchun, Northeastern China. Int. J. Environ. Res. Public Health 2017, 14, 226. [CrossRef]

35. Yu, J.; Ma, Y.; Yang, S.; Pang, K.; Yu, Y.; Tao, Y.; Jin, L. Risk Factors for Cardiovascular Disease and Their Clustering among Adults in Jilin (China). Int. J. Environ. Res. Public Health 2016, 13, 70. [CrossRef] [PubMed]

(C) 2020 by the authors. Licensee MDPI, Basel, Switzerland. This article is an open access article distributed under the terms and conditions of the Creative Commons Attribution (CC BY) license (http://creativecommons.org/licenses/by/4.0/). 